\title{
3D Modeling Parallel Coupled-Line Bandpass Filter Based on Coplanar Waveguide MMIC Multilayer Technology
}

\author{
Rizqi Eka Putri ${ }^{1}$, Emerson Pascawira Sinulingga ${ }^{2 *}$, Suherman $^{3}$ \\ ${ }_{1,2,3}$ Universitas Sumatera Utara \\ J1. Dr. T. Mansur No. 9, Medan 20155, Indonesia \\ emerson.sinulingga@usu.ac.id*
}

\begin{abstract}
Electromagnetic modeling technique on monolithic microwave integrated circuit (MMIC) coplanar waveguide (CPW) multilayer have been developed to accurately model the Parallel Coupled-Line Bandpass Filter. The 3D modeling technique shows the simulation results that are optimum. Several simulation steps have been demonstrated and compared on the design of Parallel Coupled-Line Bandpass Filters. Based on the 3D modeling, S11-Return Loss and S21-Insertion Loss of -22.6 dB and and $2.94 \mathrm{~dB}$ are obtained respectively. In addition, it is shown the best frequency response from the design of the Parallel Coupled-Line Bandpass Filter.
\end{abstract}

Keywords: Electromagnetic modeling, Monolithic Microwave Integrated Circuit (MMIC), 3D modeling, Coplanar Waveguide (CPW), and Parallel Coupled-Line Bandpass Filter.

\section{Introduction}

The Monolithic Microwave Integrated Circuit (MMIC) technology develops very rapidly in cellular mobile communication. This is due to its features such as component miniaturization, lightness, good reproducibility with large production capabilities and high frequency applications [1]-[3]. There are several publications [3]-[5] which show simulation results that were good agreement with the measurement results in realizing MMIC technology. However, the use of the features of the 3D electromagnetic modeling is not fully exploited because RF/Microwave designers rely mostly on the default simulation parameters. There are several important features that can increase the accuracy of layout modeling such as "reference offset, mode of operation, and mesh formats. In the work published in [6], it is shown the effectiveness of utilizing these features in designing passive and active components. Therefore in this paper, it is demonstrated further the use of accurate 3D modeling technique in optimizing the design and simulation of Parallel Coupled-Line Bandpass Filter that has operating frequency of $2 \mathrm{GHz}$. This design has been realized using multilayer Coplanar Waveguide in MMIC environment.

\section{Research Method}

There are several simulation steps in designing MMIC using simulators such as Agilent ADS Momentum $\odot$ which are suitable for designing layout components i.e. defining substrate, settingup ports, defining frequency and output plans as well as defining mesh simulation [6]. The followings are important parameters that need to be taken into account for accurate layout simulation. The first simulation parameter to be taken in to account is the importance of placing a reference offset in the input and output of the component layout. Reference offset is the position of the probe which is very important with respect to the probe pads. As illustrated in Figure 1, several simulations have been carried-out with a reference offset of default $0,30,60,90$ microns. It is demonstrated that the most accurate reference offset for the probe must be in between 30 to 60 microns (middle) of the probing pads of the layout design.

TELKA, Vol.5, No.1, Mei 2019, pp. 24 30

ISSN (e): 2540-9123

ISSN (p): 2502-1982 


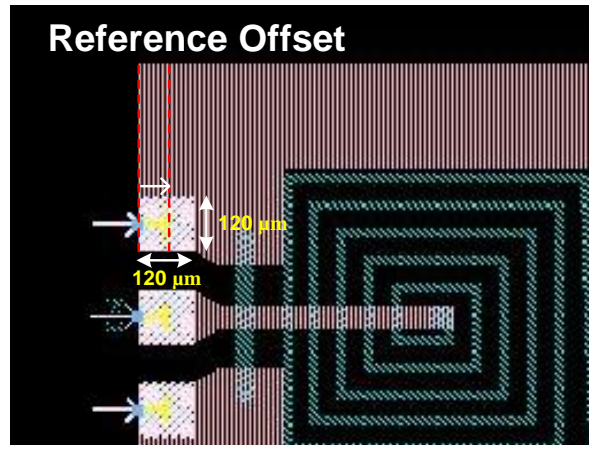

Figure 1. Top-view of probing pads reference offset.

The second important simulation parameter is to determine the conductor layer property in the substrate editor. There are two important simulation parameters in the conductor layer property which are the material and the operation conductor layer. The default simulation parameter of a conductor material is perfect conductor, whereas in fabricating components it is impossible for a perfect conductor material to be obtained. Therefore, in designing the material gold is used and set-up to be accurately to model the fabrication process. Gold is a type of conductor with a very good conductivity. Component characteristic losses resulting from default simulation settings do not represent accurate losses that occur in the fabricated component in onwafer measurements [6]. This is caused by the laying operation of the conductor layer in the default simulation parameters. In default simulation settings, the loss of conductor has not been considered [6]. The solution is to expand the conductor layer using the 3D expansion feature by selecting operation conductor as intrude into substrate with the above interface position between the metal conductor and the substrate.

Mesh density is the number of elements per unit area in a mesh for accurate analysis results. As the number increases, the computation or analysis will take a longer time. The default simulation parameter in mesh density is 20 cells/wavelength. Mesh density applied to the simulation allows the simulator to calculate the currents in each cell leading to the calculation of component S-parameters [6]. In this research, several settings to get the optimum mesh density (simulations with mesh density: 10, 20, 30, 40 cells/wavelength) have been carried-out. The higher the mesh density value, the longer the simulator works. In this work, the optimum mesh density with regards to the simulation time is 30 cells/wavelength. The summary of the steps simulation is shown in Table 1 below.

Table 1. Simulation steps in 3D Electromagnetic Modelling of MMIC components.

\begin{tabular}{ccccc}
\hline Simulation & $\begin{array}{c}\text { Reference } \\
\text { Offset }\end{array}$ & Material & Operation & $\begin{array}{c}\text { Mesh } \\
\text { Density }\end{array}$ \\
\hline Step1 & 0 & Perfect Conductor & Sheet & 20 \\
Step2 & 60 & Perfect Conductor & Sheet & 20 \\
Step3 & 60 & Gold & Sheet & 20 \\
Step4 & 60 & Gold & Intrude & 20 \\
Step5 & 60 & Gold & Intrude & 30 \\
\hline
\end{tabular}

\section{Results and Analysis}

Bandpass filter is a type of filter that passes signals that range in frequency from the lower frequency (cut-off frequency low $/ f_{1}$ ) to the upper limit frequency (cut-off frequency high $/ f_{2}$ ). The cut-off frequency $\left(f_{c}\right)$ is the frequency that becomes the limit for passing or blocking the input signal. In Figure 2 it is presented clearly the typical of the bandpass filter frequency response. In 
this response, the characteristics of MMIC components are expressed in S-Parameters such as $\mathrm{S}_{11}$-Return Loss and $\mathrm{S}_{21}$-Insertion Loss. Return Loss (RL) is the ratio of the power received with the power delivered. In the $S$-parameter, it is called $S_{11}$, which is the loss of signal strength due to reflection in the network, which results from the impedance of the transmission line. In a transmission, the maximum reflected power allowed is $10 \%$ lost in the receiver. Then the maximum RL value is $10 \mathrm{~dB}$, it can be calculated using equation "(1)". $P_{R}$ is the reflected power and $P_{T}$ is the transmitted power,

$$
R L(d B)=10 \log \frac{P_{R}}{P_{T}}
$$

Insertion Loss (IL) is a loss of power due to the insertion of a tool between the source and the load (measuring instrument). The $\mathrm{S}$-parameter is represented by $\mathrm{S}_{21}$, which is the measure of the loss of signal power in a circuit.

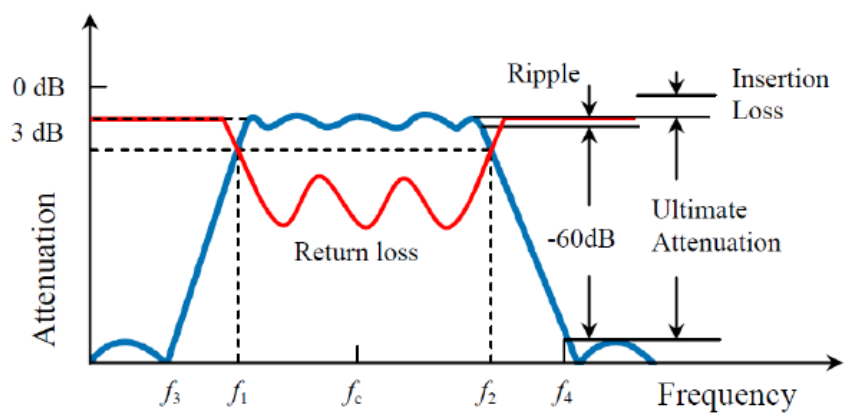

Figure 2. Typical bandpass filter response [7].

In this research, the accurate 3D electromagnetic modelling were carried-out for designing MMIC parallel coupled-line bandpass filter. Figure 3a shows the design of a parallel coupled-line bandpass filter component that is designed to operate at a frequency of $2 \mathrm{GHz}$. The MMIC modeling utilises multilayer CPW technique with Gallium Arsenide (GaAs) as the semi insulating substrate with thickness of 600 microns. The multilayer composition consists of five layers with three layers of metal conductors coded with M1, M2 and M3 with a thickness of 0.8 microns, and two dielectric layers, PI1 and PI2 with a thickness of 2.5 microns as presented in its cross-sectional view in Figure $3 b$.

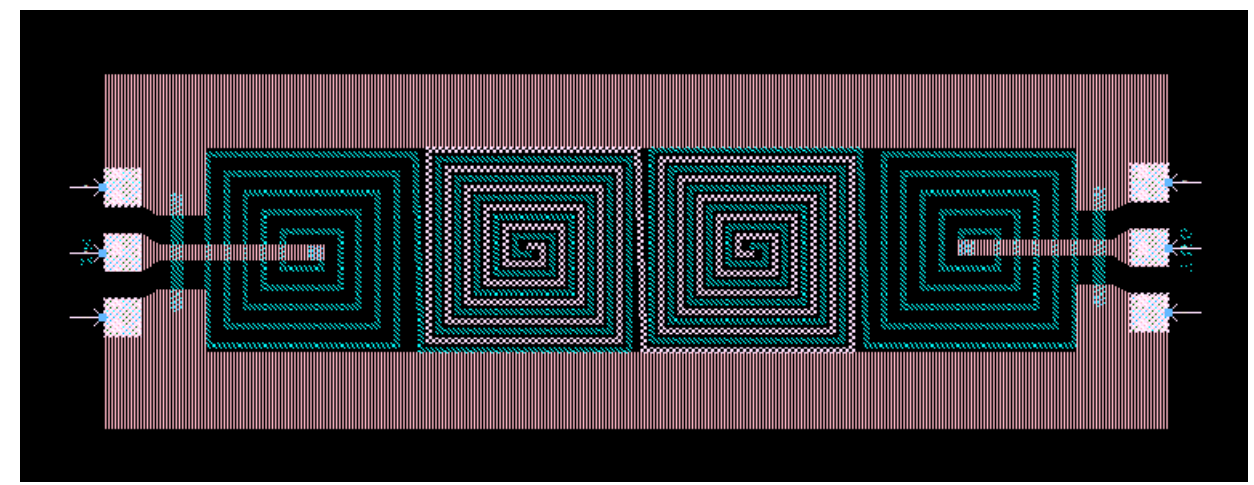

(a) 


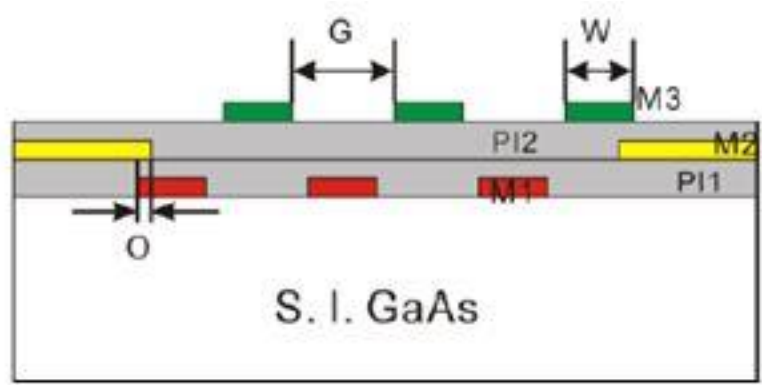

(b)

Figure 3. Design of multilayer CPW of (a) MMIC parallel coupled-line bandpass filter component, and (b) its cross sectional view.

Figure 4 and Figure 5 are the graphs of the $S_{11}$-Return Loss and $S_{21}$-Insertion Loss simulation of the parallel coupled-line bandpass filter component using 3D modeling simulation steps [6]. In the graphs of the $S_{11}$-Return Loss and $S_{21}$-Insertion Loss, there is a very significant difference in the step changes in the simulation.

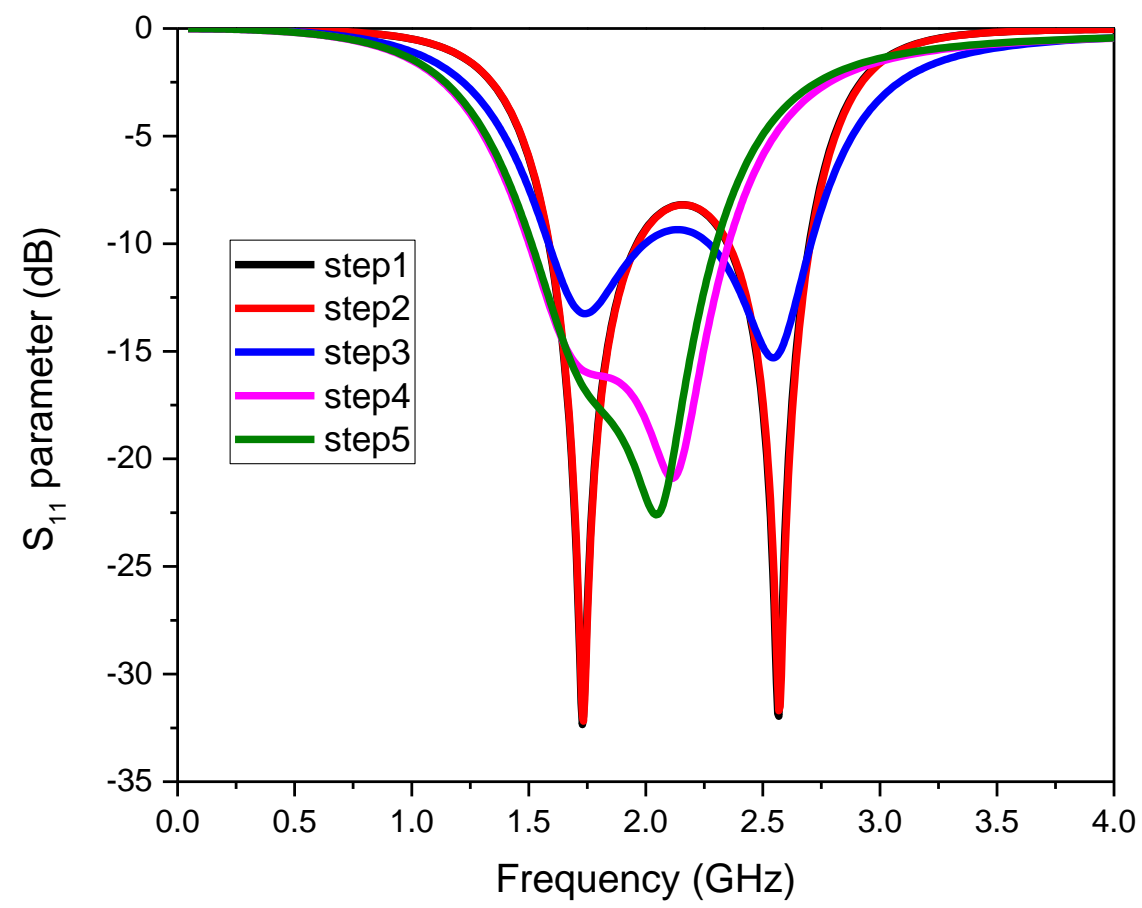

Figure 4. Graphs of $S_{11}$-Return Loss parallel coupled-line bandpass filter.

The bandpass filter responses from the optimum simulation are shown in Figure 6 and 7. By utilizing the accurate $3 \mathrm{D}$, the frequency cut-off occurs at $f_{c}=1.83 \mathrm{GHz}$. From the simulation its is shown that the low frequency cut-off starts at $f_{l}=1.25 \mathrm{GHz}$ and the frequency cut-off high occurs at $f_{2}=2.41 \mathrm{GHz}$. The Parallel Coupled-Line Bandpass Filters has the return loss value $\mathbf{S}_{11}$ $=-22.6 \mathrm{~dB}$ and the value of insertion loss $\mathrm{S}_{21}=2.94 \mathrm{~dB}$ at $2 \mathrm{GHz}$. 


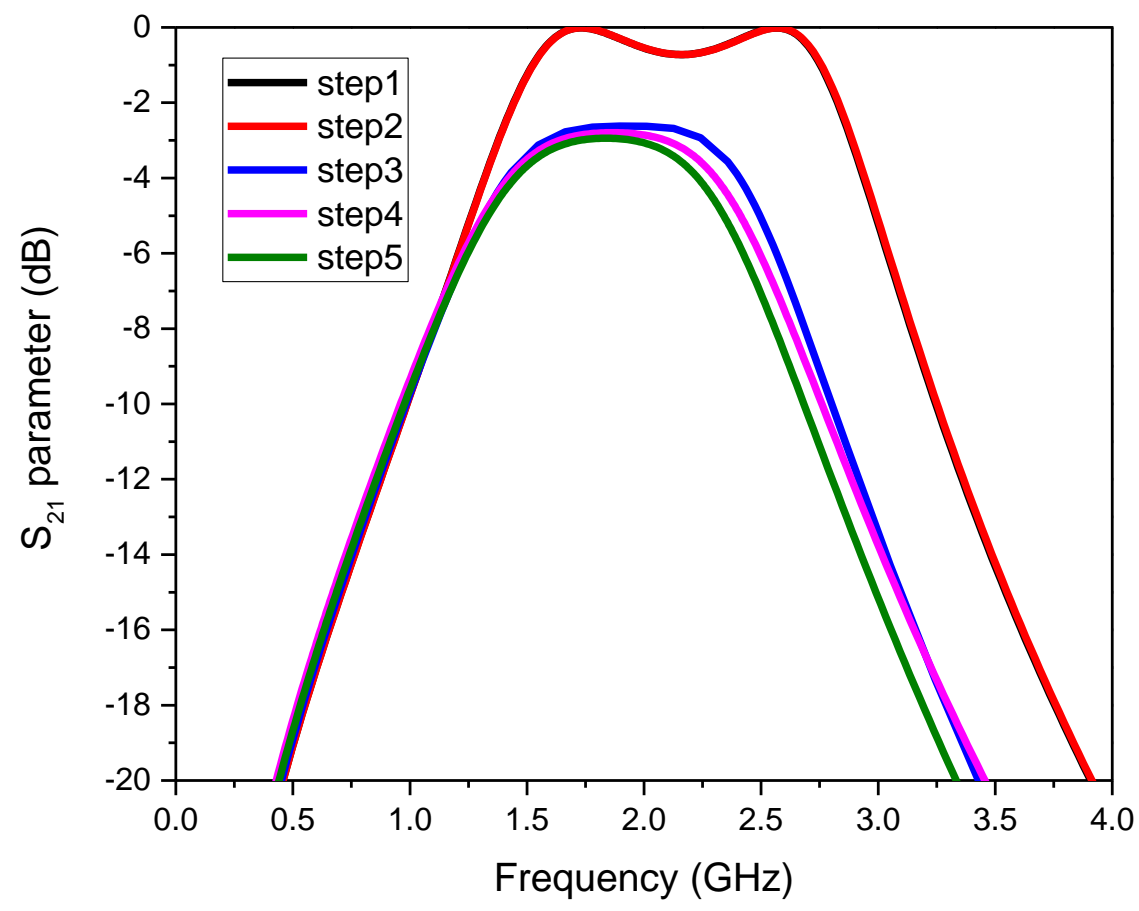

Figure 5. Graphs of $S_{21}$-Insertion Loss parallel coupled-line bandpass filter.

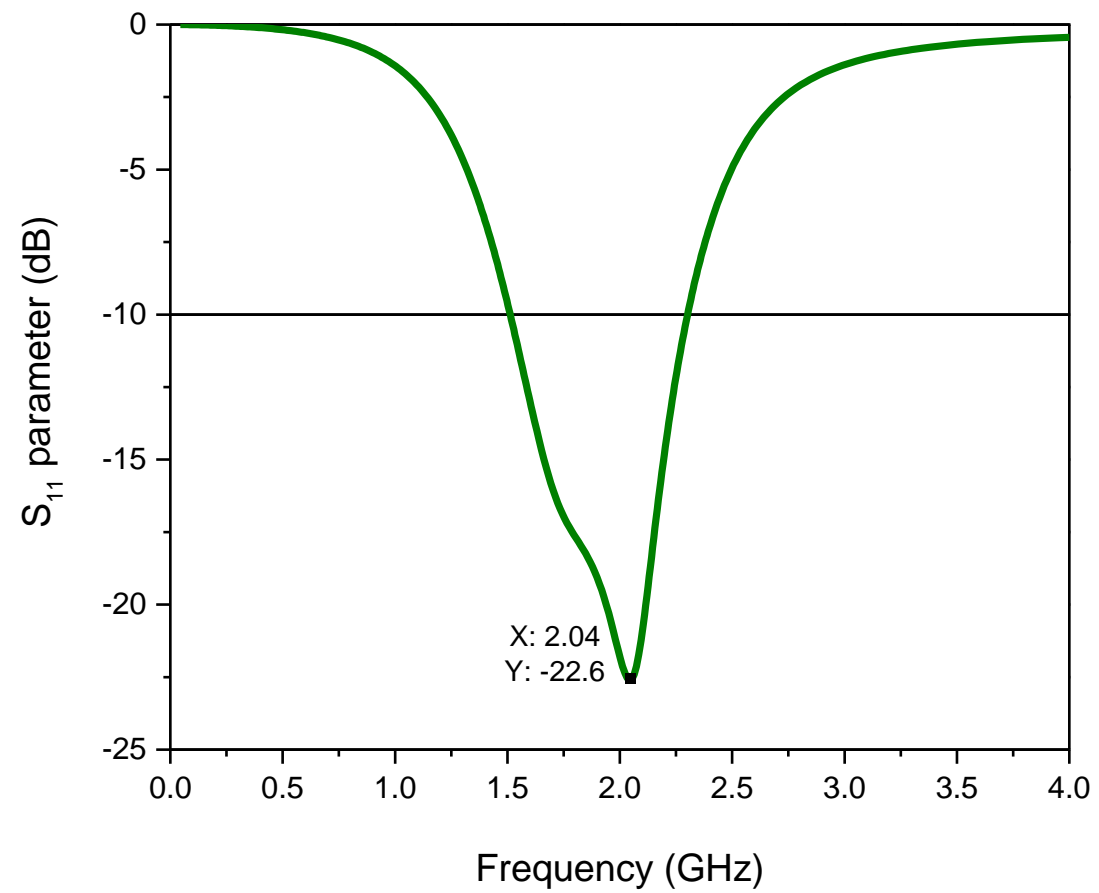

Figure 6. Graphs filter response of $S_{11}$-Return Loss parallel coupled-line bandpass filter. 


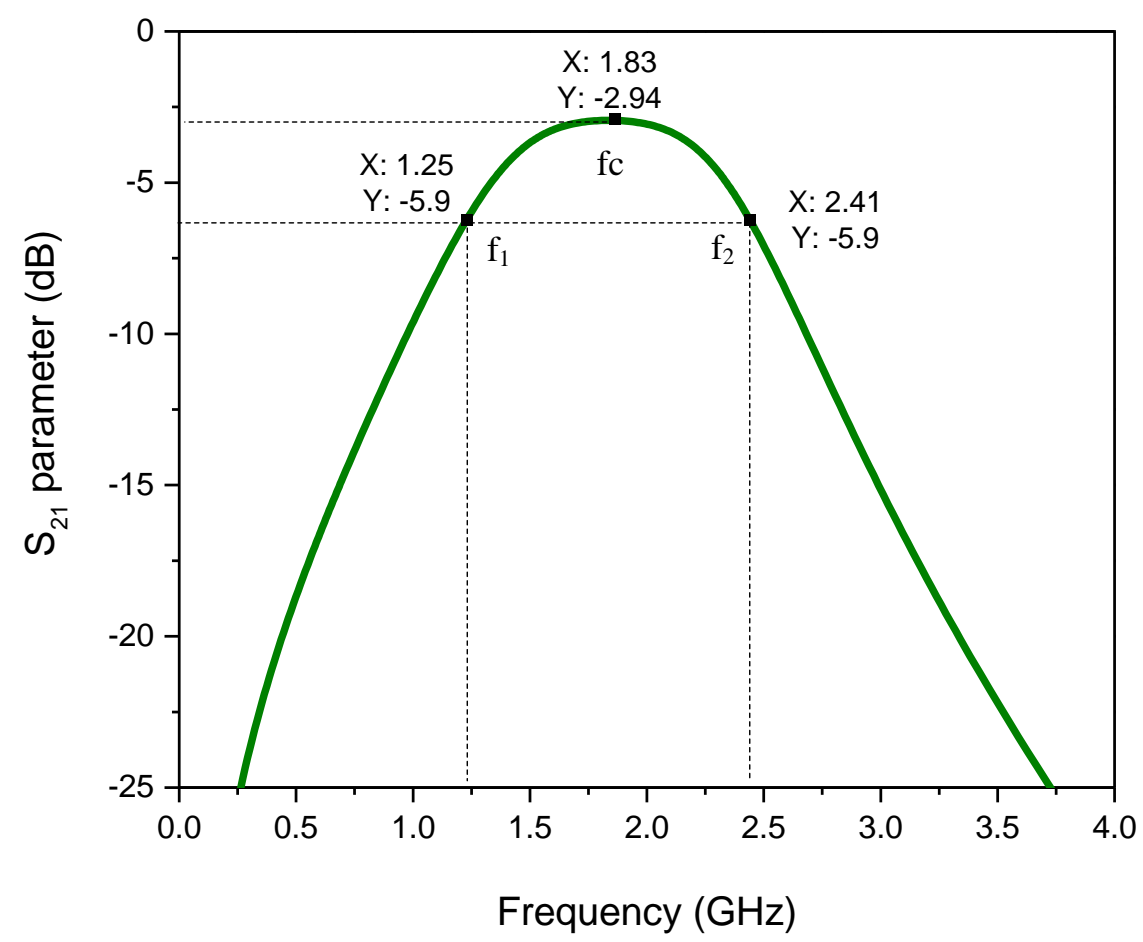

Figure 7. Graphs filter response of $S_{21}$-Insertion Loss parallel coupled-line bandpass filter.

\section{Conclusion}

Utilizing accurate simulation parameters and settings are very important to increase the efficiency of multilayer MMIC components. Determining optimum simulation parameters will make it easier for designers to accurately design components before fabrications In this works it has shown that Return Loss of $S_{11}=-22.6 \mathrm{~dB}$ and Insertion Loss of $S_{21}=2.94 \mathrm{~dB}$ has been obtained accurately for the operating frequency of $2 \mathrm{GHz}$.

\section{References}

[1] S. Marsh, "Practical MMIC Design," London: Artech House Inc, 2006.

[2] R. A. Pucel, "Design Considerations for Monolithic Microwave Circuits," Microwave Theory and Techniques, IEEE Transactions On, vol. 29, pp.513-534, 1981.

[3] I. D. Robertson and S. Lucyszyn, "RFIC and MMIC Design and Technology," The Institution of Electrical Engineers, 2001.

[4] Q. Sun, V. T. Vo, J. Tan, R. A. Davies and A. A. Rezazadeh, "Miniaturized Multilayer CPW pHEMT Amplifiers," in European Microwave Integrated Circuit Conference, 2008 IEEE 3rd, pp. 44-47, 2008.

[5] Momentum 3D Planar EM Simulator. Cited 2019; Available from: https://www.keysight.com/en/pc-1887116/momentum-3d-planar-emsimulator? $\mathrm{cc}=\mathrm{ID} \& \mathrm{lc}=\mathrm{eng}$.

[6] Emerson P. Sinulingga, A. A. Rezazadeh and Peter B. K. Kyabaggu, "3D Momentum Modeling Technique and Measurements of CPW Compact GaAS Multilayer MMICs," IEEE, 2015.

[7] A. Alburaikan, "Metamaterial Structure Inspired Miniature," Ph. D thesis, Manchester, UK: The University of Manchester, 2016. 
[8] Q. Sun, J. Yuan, J. Lu, A. A. Rezazadeh, L. Krishnamuthy and V. T. Vo, "Compact Multilayer CPW MMIC Spiral Directional Coupler and Bandpass Filter," in IEEE, Paris, France, 2010.

[9] R. W. Jackson, "Considerations in the use of Coplanar Waveguide for Millimeter-Wave Integrated Circuits," Microwave Theory and Techniques, IEEE Transaction On, vol. 34, pp. 1450-1456, 1986.

[10] C. P. Wen, "Coplanar Waveguide: A Surface Strip Transmission Line Suitable for NonReciprocal Gyromagnetic Device Application," Microwave Theory and Techniques, IEEE Transactions On, vol. 17, pp. 1087-1090, 1969.

[11] M. P. David, "Microwave Engineering," United States of America: JohnWiley \& Sons, Inc, 2012.

[12] I. Bahl, "Lumped Elements for RF and Microwave Circuits," London: Artech House, 2003. 